\title{
Coordenação dos cuidados em saúde pela atenção primária à saúde e suas implicações para a satisfação dos usuários
}

Health care coordination by primary health care and its implications for users' satisfaction

Patty Fidelis de Almeida', Lígia Giovanella², Berardo Augusto Nunan³

' Doutora em Saúde Pública pela Escola Nacional de Saúde Pública Sergio Arouca, Fundação Oswaldo Cruz (ENSP/FIOCRUZ) - Rio de Janeiro (RJ). Professora do Centro de Ciências da Saúde, Universidade Federal do Recôncavo da Bahia (UFRB) - Cruz das Almas (BA), Brasil.

patty@ufrb.edu.br

2 Doutora em Saúde Pública pela ENSP/FIOCRUZ. Pesquisadora Titular da ENSP/FIOCRUZ - Rio de Janeiro

(RJ), Brasil.

giovanel@ensp.fiocruz.br

${ }^{3}$ Especialista em Políticas Públicas e Gestão Estratégica em Saúde pela Escola Nacional de Administração Pública (ENAP). Médico da Fundação de Ensino e Pesquisa em Ciências da Saúde da Secretaria de Estado de Saúde - Brasília (DF), Brasil. berardo.sesdf@gmail.com
RESUMO $O$ presente artigo analisou as possíveis relações entre a coordenação dos cuidados pela Atenção Primária à Saúde e a satisfação dos usuários nos municípios de Aracaju, Belo Horizonte, Florianópolis e Vitória. Indicadores selecionados para avaliar duas dimensões da coordenação - integração entre níveis assistenciais e fortalecimento da Atenção Primária à Saúde - foram confrontados com aqueles de avaliação da satisfação dos usuários com o desempenho das equipes de Saúde da Família. Os resultados apontam que as mudanças empreendidas pela Estratégia Saúde da Família se refletem na satisfação geral com a Atenção Primária à Saúde, indicando melhorias quando comparadas às ações desenvolvidas por serviços 'tradicionais'.

PALAVRAS-CHAVE: Atenção Primária à Saúde; Estratégia Saúde da Família; Coordenação; Satisfação.

\begin{abstract}
The present article analyzed the possible relationships between care coordination by the Primary Health Care and users' satisfaction in four towns in Brazil - Aracaju, Belo Horizonte, Florianópolis, and Vitória. Some indicators selected to evaluate two dimensions of coordination - integration among care and strengthening levels of the Primary Health Care were compared with those related to users' satisfaction with Family Health team performance. Both results revealed that the changes introduced by the Brazilian's Family Health Strategy are reflected in an overall satisfaction with the Primary Health Care, thus they show improvements as compared with care provided by 'traditional' services.
\end{abstract}

KEYWORDS: Primary Health Care; Family Health Strategy; Coordination; Satisfaction. 


\section{Introdução}

A Constituição Federal Brasileira de 1988 inscreveu, pela primeira vez na história do Brasil, a saúde como direito de cidadania a ser garantida pelo Estado, mediante políticas econômicas e sociais que visem à redução do risco de doença e de outros agravos e ao acesso a açôes e serviços de saúde capazes de garantir o bemestar de sua população. Entre os muitos obstáculos para a implementação do Sistema Único de Saúde (SUS), destacou-se a conjuntura internacional desfavorável dos anos 1990. Em 1993, o Relatório do Banco Mundial, investindo em saúde, ressaltou que somente em algumas situaçóes saúde é uma responsabilidade pública (SENNA, 2002). Ainda, abordava a amortização dos efeitos das políticas de ajuste fiscal por meio do discurso de combate à pobreza. As liçóes aprendidas por este relatório foram: os pacotes de programas seletivos e focalizados devem ser universalizados para as populaçóes com baixa renda.

Foi neste contexto, politicamente desfavorável, que o Brasil assumiu a missão de mudar o modelo assistencial centrado no hospital e de responder aos agravos agudos, consolidados no período ditatorial, por meio da Atenção Primária à Saúde, com iniciativas bastante tímidas em seus primórdios. Em 1994, o Ministério da Saúde brasileiro criou o Programa Saúde da Família (PSF) mais seletivo e orientado às populaçóes de risco. As equipes formadas por um médico supostamente 'generalista', enfermeiro, auxiliar de enfermagem e agentes comunitários de saúde se responsabilizariam pela atenção de aproximadamente 4.500 pessoas de uma área adscrita. Nos primeiros anos, a implantação do PSF foi lenta. Até 1999 o país tinha pouco mais de 4.300 equipes que cobriam $15 \%$ da população, em geral em municípios de pequeno porte.

Em 1998, um incentivo financeiro per capita repassado diretamente do Governo Federal aos municípios impulsionou a expansão do programa e, na medida em que as açôes tomavam vulto em território nacional, o 'pacote básico de APS' crescia e mostrava suas potencialidades para instituir novas formas de atenção em saúde. Estudos realizados demonstravam, por exemplo, que, para cada $10 \%$ de aumento na cobertura populacional do PSF, havia uma redução média de 4,5\% na taxa de mortalidade infantil (MACINKO et al., 2006; 2007).

Atualmente, após 18 anos das primeiras equipes de Saúde da Família (SF), os números impressionam: 32.809 equipes e cobertura de 103,600 milhóes de brasileiros, segundo dados de março de 2012 (BRASIL, 2012). Os desafios também são do tamanho da extensão continental do país.

O PSF é a Estratégia SF (ESF) para marcar de forma inequívoca sua diferença em relação aos programas seletivos e ressaltar o potencial que tem uma 'APS forte' de construir novas formas de cuidado, com melhores resultados em relaçáo aos indicadores de saúde propriamente ditos, como também mais ética. Neste sentido, foi importante saber: quais atributos poderiam distinguir a ESF das propostas focalizadas de APS e potencializar sua capacidade de transformar o modelo de atenção à saúde no Brasil? Outra questão pareceu fundamental: a coordenação dos cuidados em saúde. Em contexto latino-americano, no qual historicamente concorrem diferentes visôes de APS, recente consenso entre especialistas reconhece a coordenação entre os distintos níveis assistenciais como um dos componentes essenciais de uma atenção primária abrangente (HAGGERTY et al., 2009).

Há certa concordância de que a coordenação dos cuidados é um tema pendente tanto para os países europeus, que desde os anos de 1990 vêm implementando uma série de reformas procoordenação (RICO et al., 2003; HOFMARCHER et al., 2007; CALNAN et al., 2007; GÉRVAS; RICO, 2005), quanto para os latino-americanos, cujo recente movimento de valorização e defesa de sistemas organizados por uma APS abrangente cria contexto favorável ao desenvolvimento de iniciativas que fortaleçam a provisão de atenção integral em saúde (ORGANIZAÇÃO PANAMERICANA DE SAÚDE, 2005; CONILL; FAUSTO, 2007; WHO, 2008; HAGGERTY et al., 2009). Os países da América Latina também enfrentam o desafio das mudanças no perfil epidemiológico de diversos grupos populacionais, com crescente prevalência de doenças crônicas, além de conviverem com problemas decorrentes das situaçóes social e econômica desfavoráveis e consequente persistência 
de enfermidades infectocontagiosas e causas externas, com maiores desafios para garantia da atenção integral e coordenação.

Mesmo entre países europeus, com coberturas universais por meio de seguros sociais com pagamento por procedimentos a profissionais autônomos, observase que as medidas para melhorar a coordenação dos cuidados têm sido mais lentas se comparadas àqueles com serviços nacionais de saúde, cuja função de gatekeeper é exercida pela APS (GERVÁS; RICO, 2005). Entre os países latino-americanos, a preocupação com o tema ocorre em meio ao movimento pela renovação da atenção primária (ORGANIZAÇÃO PANAMERICANA DE SAÚDE, 2005) - 'agora mais necessária que nunca' (WHO, 2008) -, em que a coordenação é compreendida como atributo necessário para responder aos problemas de saúde da população de forma integral (WHO, 2008; HAGGERTY et al., 2009).

O consentimento em torno da importância do tema coordenação para os sistemas de saúde é seguido por outro: as dificuldades teóricas para compreender e operacionalizar o conceito. A literatura reflete o dissenso até mesmo em relação a como nomeá-lo: 'coordenação entre níveis assistenciais' ou 'coordenação dos cuidados'? Na bibliografia consultada, foram encontrados os dois. Também se observa o uso indiscriminado dos termos continuidade, coordenação e integração, que se reflete nas diversas formas de mensurar tal atributo (HAGGERTY et al., 2003; NÚNEEZ et al., 2006). Em publicação brasileira, que adaptou e validou instrumento para avaliar as dimensóes essenciais da APS, definidas por Starfield (2002), coordenação é tomada como sinônimo de integração dos serviços (ALMEIDA; MACINKO, 2006). Em meio a dissensos e incertezas, adotou-se o conceito de 'coordenação dos cuidados', que é compreendido como a articulação entre os diversos serviços e açóes relacionados à atenção em saúde, de forma que, independentemente do local onde sejam prestados, estejam sincronizados e voltados ao alcance de um objetivo comum (ALMEIDA, 2010).

Considerou-se que a coordenaçáo dos cuidados é condiçâo necessária para o alcance de uma resposta integral, capaz de atender ao conjunto de necessidades em saúde dos usuários, que envolve a integração entre os níveis do sistema de saúde. Logo, uma resposta integral não poderia ser alcançada ao interior somente da APS, ainda que fortalecida e abrangente. Outra premissa adotada neste trabalho foi que os serviços de APS são os mais adequados para coordenar o percurso terapêutico do usuário, na maior parte dos episódios de adoecimento. Mas de qual atenção primária estamos nos relatando ao imputar-lhe a complexa missão de coordenar uma resposta integral em saúde? Certamente, não nos referimos à APS seletiva com oferta reduzida de açóes e restrita à atenção de primeiro nível. Somente uma que seja fortalecida em seus atributos essenciais, capaz de mobilizar apoios, recursos políticos, econômicos, financeiros e humanos, pode ser responsável pela coordenação dos cuidados (HAGGERTY et al., 2009; ALMEIDA et al., 2011).

Em trabalhos anteriores (ALMEIDA et al., 2010; 2011), foram analisadas duas dimensóes da coordenação dos cuidados: integração entre níveis assistenciais e fortalecimento da APS. O presente artigo resgatou alguns indicadores considerados mais potentes para análise das referidas dimensóes, apresentados em Almeida et al. (2010; 2011), e cotejou alguns resultados referentes à satisfação com ações empreendidas pela ESF. $\mathrm{O}$ pressuposto é que os municípios que apresentaram maiores avanços na coordenação dos cuidados, em suas dimensôes de integração e no fortalecimento da APS, apresentariam maior grau de satisfação com as açôes desenvolvidas pela ESF. Revisão de literatura realizada por Kringos et al. (2010) encontrou consistente relaçáo entre coordenação e melhor qualidade da atenção, maior eficiência e menores custos. Neste sentido, a avaliação na perspectiva dos usuários é uma das ferramentas necessárias para compreender até que ponto a atenção ofertada atende às suas expectativas em relação ao cuidado em saúde.

\section{Metodologia}

Este artigo apresenta parte dos resultados de uma pesquisa realizada em 2008, denominada "Estudos de caso sobre a implementação da ESF em grandes centros urbanos" (GIOVANELLA et al., 2009), financiada pelo Ministério da Saúde/Departamento de Atenção Básica e apoiada pelo Teasdale-Corti Global Research 
Partnership Program, financiado pela Canadian Global Health Research Initiative. Os municípios para os estudos de caso foram selecionados de forma intencional, de modo a escolher experiências consolidadas de implantação da ESF, sendo os casos Aracaju, Belo Horizonte, Florianópolis e Vitória.

No presente artigo, analisou-se a coordenação dos cuidados considerando duas de suas dimensóes (integraçâo entre níveis assistenciais e fortalecimento da APS) discutidas em dois artigos anteriores (ALMEIDA et al., 2010; 2011). Foram selecionados indicadores considerados mais potentes para analisar as dimensóes da coordenaçáo e seus resultados foram confrontados com indicadores de avaliação da satisfação dos usuários com as açóes desenvolvidas pelas equipes de SF.

As análises, desde a perspectiva dos usuários, foram realizadas baseando-se em um inquérito domiciliar, com a aplicação de questionários fechados à amostra de famílias cadastradas. O informante familiar foi o chefe da família ou cônjuge, entrevistado no domicílio. $\mathrm{O}$ plano de amostragem foi do tipo conglomerado em três estágios de seleção: equipe de SF como unidade primária de amostragem, agente comunitário de saúde (ACS) como secundária e, como elementar, a família cadastrada. Cinco famílias selecionadas aleatoriamente de cada dois ACS das equipes selecionadas foram entrevistadas. Em Vitória, em função do menor número de equipes existentes, foram aplicados questionários a três famílias de cada ACS. Foram entrevistadas 3.311 famílias em 2008 (Aracaju - 800, Belo Horizonte - 900, Florianópolis - 789 e Vitória - 822).

De forma transversal, foram analisados resultados referentes à integração e ao fortalecimento da APS, com vistas a cotejar as possíveis relaçóes entre tais dimensóes e a avaliação da satisfação dos usuários com as açóes desenvolvidas pelas equipes de SF.

As duas dimensões mencionadas para análise da coordenação dos cuidados foram descritas e avaliadas com base em informaçóes convergentes, trianguladas para responder às perguntas de investigação, buscando articular a perspectiva de gestores, profissionais das equipes e famílias cadastradas. $\mathrm{O}$ objetivo foi confrontar as ações empreendidas pelas gestóes municipais e seus possíveis resultados no acesso e na utilização dos serviços de APS por parte da populaçáo (ALMEIDA et al., 2010; 2011). O Quadro 1 apresenta dimensóes, variáveis e indicadores para análise das estratégias de fortalecimento da APS e integração entre níveis assistenciais.

O estudo foi aprovado pelo Comitê de Ética em Pesquisa da Escola Nacional de Saúde Pública Sergio Arouca, Fundação Oswaldo Cruz (ENSP/Fiocruz) e dos municípios estudados, tendo cumprido às exigências estabelecidas pela Resolução 196/96 do Conselho Nacional de Saúde.

Quadro 1. Dimensões, variáveis e indicadores para análise das estratégias de fortalecimento da Atenção Primária à Saúde e integração entre níveis assistenciais, em quatro grandes centros urbanos, 2008

\begin{tabular}{|c|c|c|}
\hline Dimensões & Variáveis & Indicadores \\
\hline & & Fortalecimento da APS \\
\hline $\begin{array}{l}\text { Posição ocupada } \\
\text { pela APS no sistema } \\
\text { de saúde }\end{array}$ & $\begin{array}{l}\text { Organização da } \\
\text { porta de entrada } \\
\text { pela APS }\end{array}$ & $\begin{array}{l}\text { Existência de porta de entrada pela APS; busca da USF como serviço de procura } \\
\text { regular }\end{array}$ \\
\hline \multirow[b]{2}{*}{ Acesso à USF } & $\begin{array}{l}\text { Conhecimento da } \\
\text { USF }\end{array}$ & Conhecimento da ESF; conhecimento do local de funcionamento da ESF \\
\hline & Acessibilidade à USF & $\begin{array}{l}\text { Facilidade de acesso ao local de funcionamento da ESF; ampliação do acesso de novas } \\
\text { parcelas da população aos serviços de saúde; acesso a consultas sem marcação prévia; } \\
\text { acesso a consultas agendadas }\end{array}$ \\
\hline
\end{tabular}




\begin{tabular}{|c|c|c|}
\hline Dimensões & Variáveis & Indicadores \\
\hline & & Fortalecimento da APS \\
\hline \multirow{7}{*}{$\begin{array}{l}\text { Capacidade de } \\
\text { resolução da APS }\end{array}$} & $\begin{array}{l}\text { Provas diagnósticas } \\
\text { solicitadas } \\
\text { diretamente pelo } \\
\text { médico da ESF }\end{array}$ & Provas diagnósticas solicitadas diretamente pelo médico da ESF \\
\hline & $\begin{array}{l}\text { Coleta de exames } \\
\text { para patologia } \\
\text { clínica na USF } \\
\end{array}$ & Existência de coleta de exames para patologia clínica na própria USF \\
\hline & $\begin{array}{l}\text { Distribuição de } \\
\text { medicamentos }\end{array}$ & $\begin{array}{l}\text { Distribuição regular de medicamentos a grupos prioritários; acesso a medicamentos } \\
\text { em atendimento realizado pela ESF }\end{array}$ \\
\hline & $\begin{array}{l}\text { Realização de } \\
\text { capacitação }\end{array}$ & Existência de capacitação continuada \\
\hline & $\begin{array}{l}\text { Supervisão das } \\
\text { equipes de Saúde } \\
\text { da Família }\end{array}$ & $\begin{array}{l}\text { Existência de profissionais de apoio às equipes de Saúde da Família; existência de } \\
\text { supervisão da equipe de Saúde da Família }\end{array}$ \\
\hline & $\begin{array}{l}\text { Atendimento aos } \\
\text { grupos prioritários }\end{array}$ & Acompanhamento aos grupos prioritários \\
\hline & $\begin{array}{l}\text { Avaliação geral } \\
\text { da capacidade de } \\
\text { resolução da ESF }\end{array}$ & $\begin{array}{l}\text { Encaminhamento para especialista; procura por hospitais e especialistas e serviços de } \\
\text { urgência após implantação da ESF; melhoria do atendimento após implantação da ESF }\end{array}$ \\
\hline $\begin{array}{l}\text { Descentralização das } \\
\text { ações e programas } \\
\text { de saúde pública/ } \\
\text { coletiva para as } \\
\text { unidades de APS }\end{array}$ & $\begin{array}{l}\text { Descentralização } \\
\text { das ações e } \\
\text { programas de saúde } \\
\text { pública/coletiva } \\
\text { para as USF }\end{array}$ & $\begin{array}{l}\text { Descentralização e grau de articulação entre os serviços de APS e as demais áreas de } \\
\text { saúde pública/vigilância à saúde }\end{array}$ \\
\hline $\begin{array}{l}\text { Reconhecimento } \\
\text { profissional e social } \\
\text { em APS }\end{array}$ & $\begin{array}{l}\text { Reconhecimento } \\
\text { profissional e social } \\
\text { em APS }\end{array}$ & Reconhecimento profissional e social em APS \\
\hline & & Integração entre níveis assistenciais \\
\hline \multirow{2}{*}{$\begin{array}{l}\text { Estrutura } \\
\text { organizacional e } \\
\text { administrativa do } \\
\text { setor de regulação } \\
\text { da rede de serviços } \\
\text { de saúde }\end{array}$} & $\begin{array}{l}\text { Organização de } \\
\text { regionais ou distritos } \\
\text { de saúde }\end{array}$ & Existência de regionais/distritos de saúde \\
\hline & $\begin{array}{l}\text { Principal } \\
\text { propriedade dos } \\
\text { prestadores de } \\
\text { serviços de saúde } \\
\end{array}$ & Propriedade dos prestadores públicos de saúde \\
\hline \multirow[t]{2}{*}{$\begin{array}{l}\text { Instrumentos de } \\
\text { integração dos } \\
\text { serviços de saúde }\end{array}$} & $\begin{array}{l}\text { Sistema de } \\
\text { regulação e } \\
\text { marcação de } \\
\text { consultas e exames } \\
\text { especializados e } \\
\text { controle de leitos e } \\
\text { internações } \\
\end{array}$ & $\begin{array}{l}\text { Existência de central municipal de regulação e marcação de consultas e exames } \\
\text { especializados; existência de central municipal de regulação e controle de leitos e } \\
\text { internações }\end{array}$ \\
\hline & $\begin{array}{l}\text { Instrumentos } \\
\text { de referência e } \\
\text { contrarreferência } \\
\text { para atenção } \\
\text { especializada }\end{array}$ & $\begin{array}{l}\text { Existência de instrumentos de referência e contrarreferência, registro de informações } \\
\text { para o serviço de referência e recebimento de contrarreferência }\end{array}$ \\
\hline
\end{tabular}




\begin{tabular}{|c|c|c|}
\hline Dimensões & Variáveis & Indicadores \\
\hline & & Integração entre níveis assistenciais \\
\hline \multirow{5}{*}{$\begin{array}{l}\text { Organização dos } \\
\text { fluxos para atenção } \\
\text { especializada, } \\
\text { hospitalar e } \\
\text { de urgência/ } \\
\text { emergência }\end{array}$} & $\begin{array}{l}\text { Percurso mais } \\
\text { comum do } \\
\text { paciente para } \\
\text { acesso à atenção } \\
\text { especializada }\end{array}$ & $\begin{array}{l}\text { Percurso mais comum do paciente desde o atendimento na USF ao encaminhamento } \\
\text { para consulta especializada; principal forma de acesso ao especialista }\end{array}$ \\
\hline & $\begin{array}{l}\text { Análise dos } \\
\text { encaminhamentos } \\
\text { para serviços } \\
\text { especializados } \\
\text { realizados pelos } \\
\text { profissionais da APS }\end{array}$ & $\begin{array}{l}\text { Existência de prática sistemática de análise dos encaminhamentos para serviços } \\
\text { especializados realizados pelos profissionais da APS }\end{array}$ \\
\hline & $\begin{array}{l}\text { Monitoramento } \\
\text { das filas de espera } \\
\text { para atenção } \\
\text { especializada }\end{array}$ & Existência de monitoramento das filas de espera para atenção especializada \\
\hline & $\begin{array}{l}\text { Fluxo formalizado } \\
\text { para acesso à } \\
\text { atenção hospitalar }\end{array}$ & Existência de fluxo formalizado para acesso à atenção hospitalar \\
\hline & $\begin{array}{l}\text { Articulação entre } \\
\text { centros de saúde e } \\
\text { serviços de pronto } \\
\text { atendimento }\end{array}$ & Existência de articulação entre centros de saúde e serviços de pronto atendimento \\
\hline \multirow{2}{*}{$\begin{array}{l}\text { Instrumentos para } \\
\text { continuidade } \\
\text { informacional }\end{array}$} & $\begin{array}{l}\text { Prontuários } \\
\text { eletrônicos }\end{array}$ & Existência de prontuário eletrônico \\
\hline & Protocolos clínicos & Existência de protocolos clínicos \\
\hline
\end{tabular}

APS: Atenção Primária à Saúde; USF: Unidade de Saúde da Família; ESF: Equipes Saúde da Família.

Fonte: adaptado de Almeida et al. (2010; 2011).

\section{Resultados e Discussão}

Fortalecimento da Atenção Primária à Saúde e integraçáo entre níveis assistenciais: atributos de uma Atençáo Primária Integral à Saúde

A seguir será apresentada uma síntese das discussóes sobre as estratégias de coordenação dos cuidados, com base em duas dimensóes: integração entre níveis assistenciais e fortalecimento da APS, bem como uma seleção de indicadores considerados mais potentes para avaliá-las.

As ações empreendidas nos municípios estudados para dar protagonismo à APS incluíram o fortalecimento de alguns de seus atributos tradicionais, como acessibilidade e porta de entrada, e outros, como resolutividade, reconhecimento profissional e social e descentralização das açóes de saúde coletiva (ALMEIDA et al., 2011). Para inserir a ESF à rede de serviços, condição essencial para a oferta de atenção integral em saúde, as cidades pesquisadas investiram no fortalecimento da capacidade regulatória nas Secretarias Municipais de Saúde, regionais e centros de saúde, organização de fluxos para atenção especializada e hospitalar e, de forma mais incipiente, para a rede de urgência e emergência, e também em instrumentos para continuidade informacional, como prontuários eletrônicos e protocolos clínicos (ALMEIDA et al., 2010).

O retrato oferecido pela pesquisa mostra que Belo Horizonte e Vitória apresentaram sistematicamente melhores resultados na avaliação de profissionais e usuários, tanto referente ao primeiro conjunto de medidas procoordenação, qual seja, 'fortalecimento da APS', com melhores resultados no acesso e na utilização segundo a população cadastrada, quanto em relação à 'integração entre níveis assistenciais' também com resultados mais favoráveis quanto ao percentual de usuários que visitaram o especialista por meio de encaminhamento da Unidade de SF (USF) e aos menores tempos 
Tabela 1. Resultados selecionados: integração entre níveis assistenciais segundo médicos, enfermeiros e famílias, equipes de Saúde da Família, em quatro grandes centros urbanos, 2008

\begin{tabular}{|c|c|c|c|c|}
\hline Indicadores & Aracaju & Belo Horizonte & Florianópolis & Vitória \\
\hline \multicolumn{5}{|c|}{ Instrumentos de integração dos serviços de saúde } \\
\hline $\begin{array}{l}\text { \% de famílias cadastradas } \\
\text { encaminhadas por profissional } \\
\text { da ESF, que relataram receber } \\
\text { informações por escrito para } \\
\text { entregar ao serviço ou especialista } \\
\text { de referência }\end{array}$ & $\begin{array}{c}n=346 \\
65,3\end{array}$ & $\begin{array}{c}n=582 \\
77,0\end{array}$ & $\begin{array}{c}n=469 \\
54,4\end{array}$ & $n=468$ \\
\hline $\begin{array}{l}\% \text { de médicos que referiram fazer } \\
\text { registros no prontuário após cada } \\
\text { consulta }\end{array}$ & 100,0 & 100,0 & 100,0 & 100,0 \\
\hline $\begin{array}{l}\text { \% de médicos que recebem a } \\
\text { contrarreferência sempre/na } \\
\text { maioria das vezes, após consulta } \\
\text { do usuário com especialista }\end{array}$ & 5,4 & 2,8 & 11,5 & 11,4 \\
\hline \multicolumn{5}{|c|}{ Organização dos fluxos } \\
\hline $\begin{array}{l}\text { Principal forma de acesso ao } \\
\text { especialista informada por } \\
\text { usuários referenciados nos } \\
\text { últimos } 12 \text { meses }\end{array}$ & $\mathrm{n}=303$ & $n=324$ & $n=227$ & $\mathrm{n}=330$ \\
\hline $\begin{array}{l}\text { Consulta com especialista foi } \\
\text { agendada pela USF }\end{array}$ & 43,2 & 66,7 & 40,1 & 62,7 \\
\hline $\begin{array}{l}\text { Procurou por conta própria sem } \\
\text { qualquer encaminhamento }\end{array}$ & 25,7 & 19,4 & 15,0 & 19,4 \\
\hline \multicolumn{5}{|l|}{$\begin{array}{l}\% \text { de médicos que conseguem } \\
\text { realizar sempre/na maioria das } \\
\text { vezes agendamento para outros } \\
\text { serviços }\end{array}$} \\
\hline Serviços de atenção especializada & 42,9 & 80,6 & 47,6 & 71,5 \\
\hline $\begin{array}{l}\text { Serviço de apoio diagnóstico e } \\
\text { terapia }\end{array}$ & 37,5 & 55,5 & 42,7 & 42,9 \\
\hline Maternidade & 57,2 & 86,1 & 75,4 & 74,3 \\
\hline $\begin{array}{l}\text { \% de usuários referenciados nos } \\
\text { últimos } 12 \text { meses pelo médico } \\
\text { da ESF que conseguiram realizar } \\
\text { os exames solicitados }\end{array}$ & $\begin{array}{c}n=272 \\
86,0\end{array}$ & $\begin{array}{c}n=303 \\
93,1\end{array}$ & $\begin{array}{c}n=174 \\
78,7\end{array}$ & $\begin{array}{c}n=260 \\
90,0\end{array}$ \\
\hline $\begin{array}{l}\text { Tempo de espera pelo } \\
\text { atendimento com o especialista } \\
\text { estimado por usuários } \\
\text { referenciados }\end{array}$ & $n=163$ & $n=241$ & $n=162$ & $n=231$ \\
\hline Até 30 dias & 59,5 & 58,1 & 43,9 & 76,5 \\
\hline 3 meses ou mais & 9,2 & 17,0 & 16,0 & 10,8 \\
\hline \multicolumn{5}{|l|}{$\begin{array}{l}\text { Tempo de espera estimado } \\
\text { por usuários referenciados por } \\
\text { médico da ESF }\end{array}$} \\
\hline Realização dos exames & $n=234$ & $\mathrm{n}=282$ & $\mathrm{n}=137$ & $n=234$ \\
\hline De 0 a 7 dias & 18,0 & 53,6 & 19,0 & 50,4 \\
\hline Em até 30 dias & 68,4 & 89,4 & 57,7 & 90,6 \\
\hline
\end{tabular}

USF: Unidade de Saúde da Família; ESF: Equipes Saúde da Família.

Fonte: adaptado de Almeida et al. (2010). 
de espera (Tabelas 1 e 2). Ressalta-se que esta análise deve ponderar o fato de que Belo Horizonte (com 466 equipes de SF e $67 \%$ de cobertura, em dezembro de 2007, e, em março de 2012, com 538 equipes de SF e $78 \%$ de cobertura, aproximadamente 1.856 milhóes de habitantes) enfrenta o desafio de ser uma grande metrópole, se comparada à Vitória (com 59 equipes de SF e 64\% de cobertura, em março de 2008 , e, em março de 2012, com 76 equipes de SF e $80 \%$ de cobertura, cerca de 262.000 habitantes) (BRASIL, 2012).

É interessante observar que Belo Horizonte apresentou melhores resultados com relação às estratégias de integração da rede, principalmente no que se refere à capacidade regulatória, com estabelecimento de contratos com o setor privado de acordo com as necessidades do SUS e descentralização das funções de regulação assistencial para os distritos e equipes de SF. A consulta com especialista foi agendada pela USF em 67\% dos casos em tal município. Embora possa aparentar baixa proporção, isso aponta para uma importante mudança em processo, que enfrenta grandes obstáculos relacionados, por um lado, ao reconhecimento do papel e importância da APS para o sistema de saúde e, por outro, a relaçôes de poder instituídas, nas quais a SF passa a comandar a agenda dos serviços de referência.

Além disso, considerando que qualquer mecanismo de coordenação dos cuidados seria incapaz de promover a oferta de atenção integral em saúde diante de uma oferta insuficiente de serviços em outros níveis, também se observou expansão dos serviços próprios municipais, principalmente especializados. A implantação de Centros de Especialidades Médicas foi uma das açóes para o aumento da oferta especializada, considerada o 'grande gargalo do SUS', segundo gestores, e, ao mesmo tempo, estratégia para oferta de acesso mais oportuno e aproximação entre especialistas e equipes de SF. Belo Horizonte apresentou o maior percentual de médicos que relataram conseguir agendar serviços especializados, de apoio à diagnose e terapia e maternidade sempre e na maioria das vezes. Mais de 90\% dos usuários referenciados nos últimos 12 meses pelo médico da equipe de SF relataram conseguir realizar os exames solicitados (ALMEIDA et al., 2010), como observado na Tabela 1. Considerando que a maioria dos problemas de saúde abordados pela APS não se caracteriza como urgências e parte significativa está relacionada a condições crônicas de saúde, a realização de exames e consultas referenciadas no prazo de até 30 dias se apresenta como resposta satisfatória pelo sistema de saúde, que permite acompanhamento e seguimento adequados dos usuários.

O estudo mostrou que, nos casos estudados, a preocupação em expandir a cobertura da ESF veio seguida de iniciativas para fortalecê-la e integrá-la à rede de serviços de saúde (GIOVANELLA et al., 2009). Como discutido, esforços foram empreendidos para definição de fluxos e para o desenvolvimento de instrumentos de integração. Ainda assim, a contrarreferência parece ser um problema histórico e de difícil resolução, com importantes implicações na coordenação. A totalidade dos médicos referiu fazer registros nos prontuários após as consultas, o que, sem dúvidas, é importante para a coordenação dos cuidados entre a equipe de SF e para a continuidade da atenção (Tabela 1). Em Vitória, 85\% dos usuários relataram receber informaçóes por escrito para entregar ao serviço ou ao especialista de referência. Apesar de ser um percentual alto, em um contexto de existência e utilização de protocolos clínicos e fluxos assistenciais implementados pelos serviços, a ausência do fornecimento de informaçóes por escrito a um serviço de referência apenas se justificaria pela existência de prontuário eletrônico integrado entre os serviços, o que estava apenas em início da implantação em Belo Horizonte e Florianópolis.

Por outro lado, em todos os casos foi muito baixo, variando de 5 a 11,5\%, o percentual de profissionais médicos que relataram receber a contrarreferência sempre ou na maioria das vezes (Tabela 1 ). Tal fato permite inferir que o prontuário eletrônico, em que está implementado, não está integrado ou não é preenchido pelos serviços de atenção especializada. Essa situação pode ser agravada também pelo significativo número de serviços contratados/conveniados ao SUS, um fator que dificulta a implementação de estratégias de integração da rede de serviços de saúde. Além disso, reforça a percepção de que os serviços de referência não reconhecem a importância e o papel da APS na integralidade da atenção, um dos motivos pelo qual não disponibilizam informaçóes necessárias à continuidade e coordenação do cuidado pelos profissionais da ESF. 
Belo Horizonte é reconhecidamente uma das experiências exitosas na implementação de mecanismos, estratégias e inovaçóes para organização do SUS. Não menos importante, é considerar seu legado institucional. Desde 1994, quando aderiu à Gestão Semiplena, o município deu início ao processo de organização de estruturas de controle e avaliação. Nos demais municípios pesquisados, essa experiência é bem mais recente, sobretudo em Florianópolis e em Vitória, cuja criação de estruturas de regulação da atençấo especializada foi impulsionada pela própria expansão da oferta de APS, por meio da ESF e pela adesão ao Pacto pela Saúde, com assinatura do termo de compromisso de gestão. Aracaju, em 1991, assumiu a Gestão Plena do Sistema Municipal, responsabilizando-se pela média complexidade e, gradativamente, pelos serviços de alta complexidade (ALMEIDA et al., 2010). Ainda, todos os municípios se sobressaem na implementação de estratégias para fortalecer as equipes de $\mathrm{SF}$, com destaque para os resultados encontrados em $\mathrm{Vi}$ tória (Tabela 2).

As ações de Saúde Pública/Coletiva se encontravam em avançado processo de descentralização para as equipes de SF, segundo avaliação de gestores e fortemente corroborada pelos profissionais médicos e enfermeiros, sendo consideradas estratégias para fortalecer a ESF em sua capacidade de coordenar o ciclo de cuidados ao interior do sistema de saúde, bem como no próprio nível da APS.

Nos quatro casos, entre as famílias que indicaram conhecer a ESF, mais de $90 \%$ consideraram de fácil acesso o local de funcionamento da unidade, o que indica diminuição de barreira geográfica de acesso. $\mathrm{O}$ município de Vitória se destaca, na avaliação dos usuários, como o que apresentou os melhores resultados para a consecução de consultas médicas, programadas e por demanda espontânea, indicando organização do processo de trabalho para acolher e vincular o usuário, sendo esta ainda uma barreira aos serviços de APS, que deveriam ter como uma de suas dimensôes essenciais o fato de ser porta de entrada 'aberta' para o sistema de saúde. Além de uma porta de entrada aberta, é necessário que também seja resolutiva. $\mathrm{O}$ acesso a medicamentos em atendimentos realizados pelas ESF foi considerado importante indicador para aumentar a resolutividade desse nível de atenção e medida de sua fortaleza. Vitória apresentou os maiores percentuais de famílias que indicaram receber os medicamentos.

É interessante observar que, embora haja diferenças quanto à percepção sobre o acesso às consultas, aos medicamentos e ao acompanhamento de grupos prioritários, resultados muito próximos foram encontrados no que se refere à avaliação das famílias quanto à resolutividade da APS. Nos quatro casos, em torno de $60 \%$ das famílias indicaram resolver o problema de saúde no atendimento prestado pelas equipes de SF, sem necessidade de encaminhamento para especialistas. Este percentual ainda está distante dos $85 \%$ de resolutividade desejado talvez indique que, além da expansão do acesso, a organização da porta de entrada para o atendimento às demandas espontânea e programada e a organização dos fluxos para os demais níveis permanecem para fortalecer a APS em um contexto brasileiro.

A insuficiente dotação tecnológica pode ser apontada como um fator que influencia a capacidade de atuação das equipes de SF, com constrangimentos sobre sua capacidade resolutiva (GERVÁS; FERNÁNDEZ, 2011). A rotatividade de profissionais, sobretudo médicos, é um problema que aparenta estar sem solução a curto prazo e que impossibilita a criação de vínculos, minimiza os resultados das capacitações empreendidas por estados e municípios e compromete a resolutividade das equipes (MEDEIROS et al., 2010). A criação de Unidades de Pronto Atendimento (UPA) também pode ser apontada como uma solução às dificuldades de atendimento à demanda espontânea, fixação de profissional médico e baixa resolutividade das equipes de SF. Um efeito adverso, além das dificuldades para integrar as UPA à rede, pode ser a concorrência por profissionais médicos, colaborando para o aumento da rotatividade nos serviços de APS.

Não menos importante é o pequeno percentual de médicos com residência ou título de especialista em Medicina de Família e Comunidade (MENDONÇA et al., 2010), fator que compromete a atuação clínica generalista (GERVÁS; FERNÁNDEZ, 2011). Estudos também apontam que equipes de SF, nas quais atuavam profissionais com residência em SF ou Medicina de Família e Comunidade, apresentaram maior alcance dos atributos da APS se comparadas àquelas sem este tipo de qualificação (LEÁO; CALDEIRA, 2011). 
Tabela 2. Resultados selecionados: fortalecimento da Atenção Primária à Saúde segundo médicos, enfermeiros e famílias, equipes Saúde da Família, quatro grandes centros urbanos, 2008

\begin{tabular}{|c|c|c|c|c|}
\hline Indicadores & Aracaju & Belo Horizonte & Florianópolis & Vitória \\
\hline \multicolumn{5}{|c|}{ Posição ocupada pela APS no sistema de saúde } \\
\hline $\begin{array}{l}\text { Famílias que indicam a USF como serviço de } \\
\text { procura regular entre aquelas com serviços } \\
\text { de procura regular }\end{array}$ & $69,6(n=610)$ & $74,4(n=765)$ & $50,3(n=586)$ & $69,1(n=621)$ \\
\hline \multicolumn{5}{|c|}{ Acesso à USF } \\
\hline $\begin{array}{l}\text { Famílias que conhecem a ESF (espontânea + } \\
\text { estimulada) }\end{array}$ & $84,1(n=800)$ & $84,4(n=900)$ & $70,0(n=789)$ & $82,5(n=822)$ \\
\hline Facilidade de acesso ao local da USF & $95,5(n=465)$ & $92,0(n=527)$ & $91,2(n=317)$ & $92,4(n=539)$ \\
\hline $\begin{array}{l}\text { Avaliação das famílias quanto ao fato de } \\
\text { conseguir consulta sem marcação prévia }\end{array}$ & $\mathrm{n}=325$ & $\mathrm{n}=389$ & $n=232$ & $n=361$ \\
\hline Muito bom/bom & 40,6 & 41,9 & 51,3 & 58,4 \\
\hline Muito ruim/ruim & 57,2 & 57,1 & 44,4 & 39,3 \\
\hline $\begin{array}{l}\text { Avaliação das famílias quanto a conseguir } \\
\text { marcar consulta médica }\end{array}$ & $n=390$ & $\mathrm{n}=475$ & $n=266$ & $n=441$ \\
\hline Muito bom/bom & 53,6 & 65,3 & 60,2 & 95,0 \\
\hline Muito ruim/ruim & 45,9 & 34,7 & 36,8 & 4,8 \\
\hline \multicolumn{5}{|c|}{ Capacidade de resolução da APS } \\
\hline $\begin{array}{l}\text { Acesso a medicamentos em atendimento } \\
\text { realizado pela ESF }\end{array}$ & $n=518$ & $\mathrm{n}=624$ & $\mathrm{n}=400$ & $\mathrm{n}=528$ \\
\hline Recebem todos & 26,6 & 36,1 & 40,5 & 45,6 \\
\hline Recebem apenas alguns & 65,5 & 60,9 & 55,3 & 50,8 \\
\hline Não recebem & 7,9 & 3,0 & 4,2 & 3,6 \\
\hline $\begin{array}{l}\text { Avaliação das famílias quanto à resolutividade } \\
\text { da ESF e necessidade de encaminhamento } \\
\text { para especialista }\end{array}$ & $\mathrm{n}=605$ & $\mathrm{n}=711$ & $\mathrm{n}=469$ & $\mathrm{n}=614$ \\
\hline $\begin{array}{l}\text { Conseguem resolver o problema de saúde } \\
\text { neste atendimento }\end{array}$ & 60,4 & 60,5 & 56,9 & 61,6 \\
\hline Precisam procurar um especialista & 35,9 & 32,5 & 39,9 & 37,5 \\
\hline Não responderam & 3,6 & 7,0 & 3,2 & 1,0 \\
\hline Acompanhamento dos hipertensos & $\mathrm{n}=397$ & $\mathrm{n}=587$ & $n=326$ & $\mathrm{n}=488$ \\
\hline USF & 58,2 & 65,4 & 53,1 & 68,6 \\
\hline Serviços privados de saúde & 25,7 & 17,4 & 20,1 & 18,9 \\
\hline Sem acompanhamento & 11,3 & 7,3 & 11,7 & 5,5 \\
\hline \multicolumn{5}{|c|}{ Descentralização das ações e programas de saúde pública/coletiva para as USF } \\
\hline $\begin{array}{l}\text { \% de médicos/enfermeiros que concordam/ } \\
\text { concordam muito que a ESF atua articulada } \\
\text { aos programas de saúde pública/coletiva }\end{array}$ & 95,5 & 92,4 & 94,7 & 90,1 \\
\hline \multicolumn{5}{|c|}{ Reconhecimento profissional e social em APS } \\
\hline $\begin{array}{l}\text { \% de médicos/enfermeiros que concordam } \\
\text { muito/concordam que os profissionais da } \\
\text { ESF desfrutam do mesmo reconhecimento } \\
\text { profissional que os especialistas }\end{array}$ & 22,0 & 13,0 & 29,4 & 14,6 \\
\hline
\end{tabular}


A pouca atração exercida pela ESF, enquanto campo de formação e atuação profissional e também frente ao imaginário social no qual prevalece a superioridade do cuidado 'o mais especializado possível', fortemente alimentado pela mídia, por corporaçóes profissionais, pela indústria farmacêutica e de equipamentos, pode ser um dos elementos que incide sobre a percepçáo dos médicos e enfermeiros quanto ao reconhecimento profissional. Curiosamente, os dois municípios que mostraram resultados mais positivos quanto à integração e ao fortalecimento da APS apresentam os mais baixos percentuais de profissionais que se percebem valorizados da mesma forma que um especialista (Tabela 2). Neste sentido, pode-se discutir também até que ponto a baixa valorização dos profissionais da APS incide sobre a importância dada à contrarreferência por parte dos profissionais que atuam nos serviços especializados.

Diante dos resultados alcançados e também dos desafios apresentados, surgem as seguintes perguntas: as estratégias de integração e fortalecimento da APS apresentadas são capazes de repercutir positivamente sobre a coordenação dos cuidados e a percepção de qualidade da atenção oferecida? Buscando responder a isso, a seguir são apresentados alguns indicadores de satisfação dos usuários com as açóes desenvolvidas pelas equipes de SF.

\section{Avaliaçáo das açóes desenvolvidas pelas equipes de Saúde da Família na perspectiva de famílias}

Foram entrevistadas 3.311 famílias nos quatro centros urbanos investigados (GIOVANELLA et al., 2009). Contudo, somente aquelas que afirmaram conhecer ou ter recebido visitas domiciliares, por qualquer integrante da equipe de SF, responderam às questóes referentes à avaliação da ESF.

Um total de 532 famílias, nas quatro cidades, que relatou haver Posto ou Centro de Saúde antes da implantação da ESF e ser atendido com frequência nestas unidades, avaliou o atendimento prestado pelas equipes de SF, comparando-as ao atendimento previamente recebido. Mais de 50\% dos entrevistados afirmou perceber melhorias no atendimento em saúde após a implantação das equipes de SF, sendo os maiores percentuais observados em Vitória e Belo Horizonte, aproximadamente $70 \%$ (Tabela 3). Também foram nestes municípios que um maior percentual de usuários afirmou procurar menos serviços de urgência/emergência, hospitais e especialistas após a implantação da ESF (ALMEIDA et al., 2011).

Açôes específicas desenvolvidas pelas equipes de SF e por Postos/Centros de Saúde preexistentes foram avaliadas pelas famílias. Em todos os sete aspectos avaliados, nos quatro casos, maior proporção de respostas apontou melhorias em relação às ações e aos atendimentos previamente recebidos. Mais de $60 \%$ dos usuários em Aracaju, Belo Horizonte e Vitória perceberam melhorias quanto à participação nas atividades do bairro, ao conhecimento dos problemas da comunidade, ao conhecimento para resolver os problemas de saúde e às condiçóes de prestar um bom atendimento (Tabela 3).

O acesso a consultas na USF foi um aspecto considerado bom/muito bom para mais de 50\% dos usuários nos quatro casos, chegando a 95\% em Vitória (Tabela 3). Se comparado à experiência prévia em Postos/Centros de Saúde, mais de 60\% dos usuários em Aracaju, Belo Horizonte e Vitória percebem haver maior facilidade para obtenção de consultas a partir da implantação da ESF. Entretanto, a facilidade para marcar consulta sem agendamento prévio foi a ação que menos concentrou respostas positivas (Tabela 3 ), confirmando outros achados que apontam dificuldades para equacionar demanda espontânea e programada nas USF (Tabela 2). Ainda sim, maior proporção de usuários referiu melhorias se comparado à experiência prévia de atendimento à demanda espontânea em Postos/Centros de Saúde.

$\mathrm{O}$ recebimento dos medicamentos prescritos apresentou-se como um obstáculo capaz de afetar a resolutividade das equipes de SF (Tabela 2). Contudo, quando comparado à experiência prévia de atendimento por unidades básicas tradicionais, cerca de 60\% dos usuários em Aracaju, Belo Horizonte e Vitória consideram que há maior facilidade para obtenção de medicamentos a partir da implantação da ESF (Tabela 3).

De modo geral, a maior parte dos usuários entrevistados está satisfeito com a ESF nos quatro grandes centros pesquisados, chegando a $74 \%$ em Vitória, município que apresenta sistematicamente resultados mais positivos em relação às estratégias para fortalecimento da APS. Tal local também apresenta o maior percentual 
Tabela 3. Indicadores de avaliação comparativa entre ações desenvolvidas pelas equipes de Saúde da Família e posto/centro de saúde previamente existente no bairro, quatro grandes centros urbanos, 2008

\begin{tabular}{|c|c|c|c|c|}
\hline $\begin{array}{l}\text { Avaliação quanto à melhoria do atendimento prestado pela ESF } \\
\text { comparado à experiência prévia de atendimento no Posto/Centro } \\
\text { de Saúde }\end{array}$ & $n=110$ & $\mathrm{n}=180$ & $n=113$ & $n=129$ \\
\hline Melhorou & 57,3 & 69,4 & 61,9 & 69,8 \\
\hline Ficou igual & 15,5 & 17,2 & 23,0 & 19,4 \\
\hline Piorou & 22,7 & 12,2 & 10,6 & 10,1 \\
\hline Não sabe & 4,5 & 1,1 & 4,4 & 0,8 \\
\hline $\begin{array}{l}\text { Avaliação quanto às ações desenvolvidas pela ESF comparadas à } \\
\text { experiência prévia de atendimento no Posto/Centro de Saúde }\end{array}$ & $n=129$ & $\mathrm{n}=180$ & $\mathrm{n}=111$ & $\mathrm{n}=129$ \\
\hline \multicolumn{5}{|l|}{ Participação nas atividades do bairro } \\
\hline Melhorou & 66,7 & 66,1 & 50,4 & 67,4 \\
\hline Ficou igual & 20,9 & 15,0 & 25,7 & 20,9 \\
\hline Piorou & 3,1 & 8,3 & 2,7 & 3,1 \\
\hline Não sabe & 9,3 & 10,6 & 19,5 & 8,5 \\
\hline \multicolumn{5}{|l|}{ Conhecimento dos problemas da comunidade } \\
\hline Melhorou & 71,3 & 68,9 & 53,1 & 72,1 \\
\hline Ficou igual & 17,8 & 15,6 & 29,2 & 17,8 \\
\hline Piorou & 3,1 & 3,9 & 2,7 & 3,1 \\
\hline Não sabe & 7,8 & 11,7 & 13,3 & 7,0 \\
\hline \multicolumn{5}{|l|}{ Conhecimento para resolver os problemas de saúde } \\
\hline Melhorou & 67,4 & 63,3 & 55,8 & 68,2 \\
\hline Ficou igual & 20,2 & 22,2 & 26,5 & 20,2 \\
\hline Piorou & 3,9 & 6,1 & 2,7 & 3,9 \\
\hline Não sabe & 8,5 & 8,4 & 13,2 & 7,8 \\
\hline \multicolumn{5}{|l|}{ Facilidade de marcar consulta } \\
\hline Melhorou & 61,2 & 66,7 & 43,4 & 62,0 \\
\hline Ficou igual & 20,2 & 17,8 & 37,2 & 20,2 \\
\hline Piorou & 14,0 & 12,2 & 6,2 & 14,0 \\
\hline Não sabe & 4,7 & 3,4 & 11,5 & 3,9 \\
\hline \multicolumn{5}{|l|}{ Facilidade de conseguir consulta sem marcação } \\
\hline Melhorou & 41,1 & 45,0 & 33,6 & 42,6 \\
\hline Ficou igual & 25,6 & 24,4 & 39,8 & 24,8 \\
\hline Piorou & 22,5 & 21,7 & 10,6 & 22,5 \\
\hline Não sabe & 10,9 & 8,9 & 14,1 & 10,1 \\
\hline \multicolumn{5}{|l|}{ Condições de prestar bom atendimento } \\
\hline Melhorou & 63,6 & 71,9 & 58,4 & 64,3 \\
\hline Ficou igual & 23,3 & 19,4 & 23,9 & 23,3 \\
\hline Piorou & 8,5 & 5,0 & 3,5 & 8,5 \\
\hline Não sabe & 4,7 & 3,9 & 12,4 & 3,9 \\
\hline \multicolumn{5}{|l|}{ Facilidade para obtenção de medicamentos } \\
\hline Melhorou & 59,7 & 57,8 & 43,4 & 60,5 \\
\hline Ficou igual & 30,2 & 27,8 & 38,9 & 30,2 \\
\hline Piorou & 3,9 & 11,7 & 3,5 & 3,9 \\
\hline Não sabe & 6,2 & 2,8 & 12,4 & 5,4 \\
\hline
\end{tabular}

ESF: Equipe de Saúde da Família.

Fonte: NUPES/DAPS/ENSP/FIOCRUZ - Pesquisa Saúde da Família quatro estudos de caso, 2008. 
Tabela 4. Indicadores de avaliação geral de satisfação com a Estratégia Saúde da Família, quatro grandes centros urbanos, 2008

\begin{tabular}{|c|c|c|c|c|}
\hline Indicadores & $\begin{array}{c}\text { Aracaju } \\
\quad \% \\
n^{*}=605\end{array}$ & $\begin{array}{c}\text { Belo Horizonte } \\
\qquad \begin{array}{c}\% \\
n=711\end{array}\end{array}$ & $\begin{array}{c}\text { Florianópolis } \\
\begin{array}{c}\% \\
n=469\end{array}\end{array}$ & $\begin{array}{c}\text { Vitória } \\
\begin{array}{c}\% \\
\mathrm{n}=614\end{array}\end{array}$ \\
\hline \multicolumn{5}{|c|}{$\begin{array}{l}\text { Avaliação das famílias quanto à } \\
\text { melhoria das condições de saúde do } \\
\text { seu bairro após a implantação da ESF }\end{array}$} \\
\hline Melhorou & 63,3 & 72,0 & 53,3 & 81,3 \\
\hline Piorou & 5,3 & 2,8 & 2,6 & 2,0 \\
\hline Ficou igual & 22,3 & 14,1 & 17,3 & 12,4 \\
\hline Não sabe/não respondeu & 9,1 & 11,1 & 26,8 & 4,3 \\
\hline \multicolumn{5}{|c|}{$\begin{array}{l}\text { Avaliação das famílias quanto à } \\
\text { melhoria do atendimento em saúde } \\
\text { após implantação da ESF }\end{array}$} \\
\hline Melhorou muito & 26,6 & 32,9 & 22,4 & 51,3 \\
\hline Melhorou um pouco & 44,5 & 40,1 & 33,5 & 28,2 \\
\hline Ficou igual & 15,1 & 15,2 & 17,5 & 12,7 \\
\hline Piorou & 5,1 & 2,8 & 2,3 & 2,3 \\
\hline \multicolumn{5}{|c|}{$\begin{array}{l}\text { Grau de satisfação das famílias com a } \\
\text { ESF }\end{array}$} \\
\hline Satisfeito & 52,9 & 62,7 & 52,0 & 74,4 \\
\hline Mais ou menos satisfeito & 28,0 & 20,0 & 24,1 & 17,6 \\
\hline Insatisfeito & 14,1 & 9,7 & 8,5 & 5,9 \\
\hline
\end{tabular}

ESF: Equipe de Saúde da Família; ${ }^{*}$ = famílias com algum integrante com experiência de atendimento pela ESF. Fonte: NUPES/DAPS/ENSP/FIOCRUZ - Pesquisa Saúde da Família quatro estudos de caso, 2008.

de famílias que declararam que o atendimento em saúde e as condiçóes de saúde do bairro melhoraram muito após a implantação da ESF. Em todos os municípios, a maioria dos usuários declarou melhorias nas condições de saúde do bairro. A frequência de usuários insatisfeitos é pequena, variando de 6\%, em Vitória, a 14\%, em Aracaju. Apenas 15\% dos usuários nos quatro municípios avaliaram que o atendimento em saúde permanece igual após a implantação da ESF.

Os resultados parecem apontar que é inequívoca a avaliação de melhorias no atendimento em saúde e também nas condiçôes de saúde do bairro após a implantação da ESF, tanto no que se refere à satisfação geral quanto àquela que compara com o atendimento previamente prestado por Postos/Centros de Saúde (Tabela 4).
Às famílias com algum integrante com experiência de atendimento, solicitou-se que apontassem livremente o que gostam e o que não gostam na ESF. Em Aracaju, das 605 com experiência de atendimento, 13\% relataram não ter qualquer queixa e gostar de tudo. Profissionais atenciosos e atendimento médico foram mencionados por maior proporção de usuários como pontos positivos (Tabela 5). Dois terços das famílias apontaram o que não gostam. Filas de espera, carência de médicos, problemas com profissionais de saúde e demora para realização e retorno dos exames foram mencionados como motivos de insatisfação, corroborando outros achados do estudo, os quais sinalizam dificuldades para agendamento de serviços de apoio à diagnose $\mathrm{e}$ terapia (Tabela 1) e para agendamento de consultas sem marcação prévia (Tabela 2). 
Em Belo Horizonte, aproximadamente metade das 711 famílias com experiência de atendimento apontou algum aspecto do qual não gosta na ESF, destacando-se a demora no atendimento, a carência de profissionais e a demora para atendimento especializado. Profissionais atenciosos e atendimento médico, assim como em Aracaju, também foram espontaneamente citados como os aspectos que os usuários mais gostam na ESF (Tabela 5).

Em Florianópolis, das 469 famílias com algum integrante com experiência de atendimento pelas equipes de SF, 48\% apontaram livremente algum aspecto do qual não gosta na ESF, destacando-se carência de profissionais, demora na realização e retorno dos exames e para marcação de consultas. Do conjunto de famílias, $46 \%$ informaram aspectos positivos na ESF com destaque para os profissionais atenciosos e o trabalho do ACS (Tabela 5).

Em Vitória, das 614 famílias com algum integrante com experiência de atendimento pela ESF, $40 \%$ mencionaram algum aspecto de que não gosta, destacandose carência de profissionais e demora para marcação de consultas. Como nos demais casos, profissionais atenciosos foi o aspecto espontaneamente mencionado que mais agrada às famílias, seguido pela visita domiciliar e o atendimento médico (Tabela 5).

Nos quatro municípios, como motivos de insatisfação, destacou-se a demora no atendimento, o que pode significar problemas para organizaçáo da demanda espontânea, conforme evidenciado pela avaliação das famílias. Conforme apresentado na Tabela 2, variou de 39 a 57\% o percentual das que avaliaram como ruim/muito ruim a possibilidade de conseguir consulta sem marcação prévia. Demora na realização de exames e consultas especializadas também corroboram a avaliação de médicos, que apontam dificuldades para agendamento de consultas especializadas e serviços de apoio à diagnose e terapia.

\section{Considerações Finais}

Pode-se afirmar que a preocupação em instituir mecanismos de integração e fortalecer a APS ao interior do sistema de saúde está presente nos quatro municípios. Porém, reconhece-se que as experiências apresentadas não podem ser generalizadas para o conjunto dos municípios brasileiros, que apresenta graus distintos de implementação e cobertura por equipes de SF. Também se destaca o fato de que os quatro casos selecionados para o estudo constituem uma amostra por conveniência, cujo objetivo foi compreender fatores facilitadores e entraves à coordenação dos cuidados, em municípios que desenvolvem práticas exitosas em APS, reconhecidas tanto pela gestão federal quanto por outros estudos realizados.

Conforme os resultados analisados em cada um dos casos, são distintos os graus de implementação dos instrumentos para integração da rede e das iniciativas para fortalecer a APS, embora os caminhos trilhados sejam semelhantes. Mudanças empreendidas pela ESF se refletem no nível de satisfação geral com a APS e indicam melhorias quando comparadas às açóes desenvolvidas por serviços 'tradicionais' previamente utilizados.

Portanto, é possível alcançar melhorias na coordenação dos cuidados e na qualidade da atenção mesmo diante de constrangimentos impostos por rotatividade profissional, formação especializada, baixo prestígio dos trabalhadores da APS, 'gargalo' na oferta de atenção especializada, entre outros desafios. A articulação das açóes e dos serviços de saúde, de forma que, independentemente do local onde estes sejam prestados, estejam sincronizados e voltados ao alcance de um objetivo comum, foi considerada atributo de uma APS abrangente e condição necessária para o alcance de uma resposta integral, que seja capaz de atender ao conjunto de necessidades em saúde dos usuários. Nesta medida, ao ser usuário-centrada, avaliaçóes positivas por parte das famílias cadastradas reafirmam as potencialidades de mudança do modelo assistencial promovidas pela ESF, ainda que constrangimentos de caráter macro imponham limites importantes.

Destacou-se que a insuficiente oferta de atenção especializada é barreira quase intransponível para a coordenação entre níveis que acarretarão sempre longos tempos de espera e impossibilidade de cuidado oportuno, por mais desenvolvidos que estejam os mecanismos de integração da rede. Nos municípios estudados, o investimento na ampliação da rede especializada própria municipal foi uma das soluçóes encontradas. A 
Tabela 5. Avaliação das famílias quanto aos aspectos que mais gostam e que menos gostam na Estratégia Saúde da Família (citação espontânea), quatro grandes centros urbanos, 2008

\begin{tabular}{|c|c|c|c|c|}
\hline Indicadores & $\begin{array}{c}\text { Aracaju } \\
\% \\
n^{*}=605\end{array}$ & $\begin{array}{c}\text { Belo Horizonte } \\
\qquad \begin{array}{c}\% \\
n=711\end{array}\end{array}$ & $\begin{array}{c}\text { Florianópolis } \\
\begin{array}{c}\% \\
n=469\end{array}\end{array}$ & $\begin{array}{c}\text { Vitória } \\
\% \\
\mathrm{n}=614\end{array}$ \\
\hline \multicolumn{5}{|l|}{ O que mais gosta no PSF } \\
\hline Profissionais atenciosos & 31,7 & 13,5 & 26,9 & 18,4 \\
\hline Visita domiciliar & 7,8 & 10,0 & 5,8 & 10,3 \\
\hline Atendimento médico & 20,3 & 13,8 & 3,2 & 9,9 \\
\hline Trabalho dos ACS & 7,4 & 4,8 & 11,9 & 8,3 \\
\hline \multicolumn{5}{|l|}{ O que não gosta na ESF } \\
\hline Demora no atendimento & 2,6 & 12,2 & 4,3 & 3,7 \\
\hline Carência de profissionais & - & 11,7 & 6,8 & 7,3 \\
\hline Demora para atendimento especializado & 1,1 & 9,4 & 2,6 & 3,1 \\
\hline Problemas com profissionais & 2,1 & 8,0 & 3,6 & 4,4 \\
\hline Insuficiência de medicamentos & 0,1 & 7,6 & 1,1 & 1,5 \\
\hline Demora na marcação de consultas & 1,8 & 6,6 & 4,9 & 6,5 \\
\hline Acolhimento/triagem & 1,7 & 5,1 & 3,8 & 3,9 \\
\hline Demora nos exames e retorno & 3,3 & 4,6 & 5,8 & 2,0 \\
\hline
\end{tabular}

PSF: Programa Saúde da Família; ESF: Estratégia Saúde da Família; ACS: agente comunitário de saúde; *n = famílias com algum integrante com experiência de atendimento pela ESF.

Fonte: NUPES/DAPS/ENSP/FIOCRUZ - Pesquisa Saúde da Família quatro estudos de caso, 2008.

reconhecida falta de profissionais em algumas especialidades médicas parece não ser um problema tão relevante por se tratar de capitais. Ainda assim, a contratação de serviços da rede privada, uma das estratégias para suprir deficiências na oferta de atenção especializada, é dificultada, segundo avaliação dos gestores e gerentes, pela remuneração oferecida pela tabela do SUS, considerada inadequada pelos prestadores (ALMEIDA et al., 2010).

Neste trabalho, além de terem sido analisados resultados, buscou-se construir uma matriz que possibilitasse a análise de componentes da coordenação dos cuidados a ser exercida pela APS. Temos clareza que outros elementos importantes para seu alcance náo foram aqui incorporados, como por exemplo, aqueles referentes à destinação dos recursos financeiros, visto ao fato de haver forte consenso de que a distribuição do financiamento seja potente indicador de prioridade conferida a determinado modelo de atenção. Entendendo que a coordenação dos cuidados envolve não apenas a articulaçáo com os demais níveis, mas ao interior da própria equipe de APS e também com outros equipamentos locais, no que seria possível denominar de coordenação vertical, reafirma-se que não foram analisadas todas as dimensôes deste conceito.

Vale ressaltar que as dimensóes da coordenação dos cuidados analisadas e consideradas condição essencial para a oferta de Atenção Primária Integral à Saúde privilegiaram a prestação de atençáo à saúde stricto senso, no que se referiu, sobretudo, à oferta de serviços e cuidados médicos. Sabe-se, como já amplamente ratificado pela literatura e pela experiência, que outras dimensóes da APS, como a participação popular, o enfoque comunitário e a intersetorialidade, são fundamentais para atuar sobre os determinantes sociais da saúde e, sem dúvida, complementam a desejada atenção primária integral á saúde.

Por fim, pode-se afirmar que a ESF se fortaleceu e está mais integrada à rede nos casos estudados, embora ainda não seja a coordenadora do ciclo de cuidados. A coordenação dos cuidados necessita ser fortalecida, pois 
é essencial para a garantia de atenção de melhor qualidade e com mais eficiência (KRINGOS et al., 2010). Todavia, as dificuldades de ofertar um cuidado coordenado na interface entre a atenção primária, especializada e hospitalar, estão presentes, também, ainda que em menor intensidade, em países como Dinamarca, Holanda ou Inglaterra, nos quais a atenção primária tem posição forte no sistema e o médico generalista exerce a função de gatekeeper (CALNAN et al., 2006; GIOVANELLA, 2006).

A insuficiente comunicação e articulação entre prestadores e profissionais, as dificuldades de oferta para atenção especializada, a ausência de cultura de colaboração, o baixo prestígio profissional e social de seus trabalhadores e os desafios que persistem para a consolidação da porta de entrada pelas USF foram algumas dificuldades levantadas como possíveis efeitos sobre a sincronização da atenção. Estabelecer objetivos comuns, de forma que o cuidado em saúde tenha o usuário como centro e seja organizado de acordo com suas expectativas pessoais e necessidades em saúde, componentes do conceito de coordenação, apontam alguns caminhos.

Portanto, por meio desta investigação, também buscou-se colaborar para o maior conhecimento teórico e prático no campo da coordenação dos cuidados, visto ser uma dimensão dos sistemas de saúde ainda pouco desenvolvida nos países da América Latina, compreendida como essencial à oferta de atenção integral em saúde. Objetivou-se também identificar e divulgar práticas exitosas, as quais, consideradas as especificidades locais, possam constituir experiências capazes de contribuir para a construção de um SUS mais equânime, de melhor qualidade e mais direcionado às necessidades de saúde da população.

\section{Referências}

ALMEIDA, C:; MACINKO, J. Validação de uma metodologia de avaliação rápida das características organizacionais e do desempenho dos serviços de atenção básica do Sistema Único de Saúde (SUS) em nível local. Série Técnica Desenvolvimento de Sistemas e Serviços de Saúde, 10. Brasília, DF: Organização Panamericana de Saúde/ Ministério da Saúde, 2006.

ALMEIDA, P.F. et al. Desafios à coordenação dos cuidados em saúde: estratégias de integração entre níveis assistenciais em grandes centros urbanos. Cadernos de Saúde Pública, Rio de Janeiro, v. 26, n, 2, 2010, p. 286-298.

ALMEIDA, P.F.; FAUSTO, M.C.R.; GIOVANELLA, L. Fortalecimento da atenção primária à saúde: estratégia para potencializar a coordenação dos cuidados. Revista Panamericana de Salud Pública, v. 29, n. 2, 2011, p. 84-95.

BRASIL. Departamento de Atenção Básica. Histórico de Cobertura da Saúde da Família. 2012. Disponível em: <http://dab.saude.gov.br/ historico_cobertura_sf.php>. Acesso em: 5 mai. 2012.

CALNAN, M.; HUTTEN, J.; TILAK, H. The challenge of coordination: the role of primary care professional in promoting integration across the interface. In: SALTMAN, R.S.; RICO, A.; BOERMA, W.G.W. (Ed) Primary care in the driver's seat? Organizational Reform in European primary care. Berkshire: Open University Press, 2007. p. 85-104.
CONILL, E.; FAUSTO, M. Análisis de la problemática de la integración de la APS en el contexto actual: causas que inciden en la fragmentación de servicios y sus efectos en la cohesión social. Rio de Janeiro: EuroSocial Salud, 2007.

GERVÁS, J.; FERNÁNDEZ, M.P. Uma Atenção Primária forte no Brasil. Relatório sobre como fortalecer os acertos e corrigir as fragilidades da Estratégia de Saúde da Família. 2011. Disponível em <http:// www.sbmfc.org.br/media/file/documentos/relatoriofinal_ portugues.pdf>. Acesso em: 5 mai. 2012.

GÉRVAS, J.; RICO, A. La coordinación en el sistema sanitario y su mejora a través de las reformas europeas de la Atención Primaria. Sociedad Española de Médicos de Atención Primaria, Madrid, v. 31, n. 9, 2005, p. 418-423.

GIOVANELLA, L. A atenção primária à saúde nos países da União Européia: configurações e reformas organizacionais na década de 1990. Cadernos de Saúde Pública, Rio de Janeiro, v. 22, n. 5, 2006, p. 951-964.

GIOVANELLA, L.; ESCOREL, S.; MENDONÇA, M.H.M. (Coord). Estudos de Caso sobre a Implementação da Estratégia Saúde da Família em Grandes Centros Urbanos. Relatório Final. Rio de Janeiro: ENSP/Fiocruz, 2009. Disponível em: <http://www4. ensp.fiocruz.br/biblioteca/home/exibedetalhesBiblioteca. 
cfm?|D=9439\&tipo=B>. Acesso em: 5 mai. 2012.

HAGGERTY, J.L. et al. Continuity of care: a multidisciplinary review. BMJ, Londres, n. 327, 2003, p. 1219-1221.

HAGGERTY, J.L.; YAVICH, N.; BÁSCOLO, E.P. Grupo de Consenso sobre un Marco de Evaluación de la Atención Primaria en América Latina. Un marco de evaluación de la atención primaria de salud en América Latina. Revista Panamericana de Salud Pública Washington, v. 26, n. 5, 2009, p. 377-384.

HOFMARCHER, M.M.; OXLEY, H.; RUSTICELLI, E. Improved health system performance through better care coordination. Paris: OECD 2007.

KRINGOS, D.S. et al. The breadth of primary care: a systematic literature review of its core dimensions. BMC Health Services Research, Londres, v. 10, 2010, p. 65.

LEÃO, C.D.A.; CALDEIRA, A.P. Avaliação da associação entre qualificação de médicos e enfermeiros em atenção primária em saúde e qualidade da atenção. Ciência \& Saúde Coletiva, Rio de Janeiro, v. 16, n. 11, 2011, p. 4415-4423.

MACINKO, J.; GUANAIS, F.C.; SOUZA, M.F.M. An Evaluation of the impact of the Family Health Program on infant mortality in Brazil, 1990-2002. Journal of Epidemiology and Community Health, Londres, v. 50, 2006, p. 13-19.

MACINKO, J. et al. Going to scale with community-based primary care: An analysis of the family health program and infant mortality in Brazil, 1999-2004. Social Science \& Medicine, 2007, doi:10.1016/j. socscimed.2007.06.028.

MEDEIROS, C.R.G. et al. A rotatividade de enfermeiros e médicos: um impasse na implementação da Estratégia de Saúde da Família. Ciência \& Saúde Coletiva, Rio de Janeiro, v. 15, 2010, p. 1521-1531.
MENDONÇA, M.H.M. Desafios para gestão do trabalho a partir de experiências exitosas de expansão da Estratégia de Saúde da Família. Ciência \& Saúde Coletiva, Rio de Janeiro, v. 15, n. 5, 2010, p. 2355-2365.

NÚÑEZ, R.T.; LORENZO, I.V.; NAVERRETE, M.L. La coordinación entre niveles asistenciales: una sistematización de sus instrumentos y medidas. Gaceta Sanitaria, Barcelona, v. 20, n. 6, 2006, p. 485-495.

ORGANIZAÇÃO PANAMERICANA DE SAÚDE. Renovação da Atenção Primária em Saúde nas Américas. Washington, DC: World Health Organization, 2005.

RICO, A.; SALTMAN, R.; BOERMA, W.G.W. Organizational Restructuring in European Health Systems: The Role of Primary Care. Social Policy \& Administration, Malden, v. 37, n. 6, 2003, p. 592-608.

SENNA, M.C.M. Equidade e política de saúde: algumas reflexões sobre o Programa Saúde da Família. Cadernos de Saúde Pública, Rio de Janeiro, v. 18, 2002, p. 203-211.

STARFIELD, B. Atenção Primária: equilíbrio entre necessidades de saúde, serviços e tecnologia. Brasília, DF: Unesco/Ministério da Saúde, 2002.

WORLD HEALTH ORGANIZATION (WHO). Primary Health Care. Now more than ever. The World Health Report 2008. Geneva: WHO, 2008.

Recebido para publicação em Julho/2012

Versão definitiva em Agosto/2012

Suporte financeiro: Ministério da Saúde/Departamento de Atenção Básica e apoiada pelo Teasdale-Corti Global Research Partnership Program.

Conflito de interesse: Inexistente 